\title{
A Proposed Framework based on Cloud Computing for Enhancing E-Commerce Applications
}

\author{
Ahmed Abou Elfetouh Saleh \\ Associative professor of Information Systems \\ Head of Information Systems Department \\ Faculty of Computers and Information, \\ Mansoura University, Egypt
}

\begin{abstract}
As a new concept, cloud computing has attracted the IT enterprise attention especially the e-commerce (EC) enterprise. At present, there is a great problem of environmental costs during the enterprises apply the ecommerce, but with the coming of cloud computing, all of the problem will be solved. Therefore, this paper will introduce a proposed e-commerce application framework based on the concepts, the origins and development trend of cloud computing which copes with the problem of ecommerce and the storage of resources. A proposed framework allows enterprises to lower costs through the effective implementation of EC activities, and solves the problem of enterprises which cannot develop e-commerce activities due to lack of resources.
\end{abstract}

\section{Keywords}

Cloud Computing, e-Commerce, e-Commerce Cloud, SaaS, IaaS, PaaS, B2B, B2C.

\section{INTRODUCTION}

E-Commerce (EC) is a popular and growing Web application which enables customers, partners and employees to achieve a Varity of purpose and services. A general definition of ecommerce, given by the Electronic Commerce Association [1, 2], is: "electronic commerce covers any form of business or administrative transaction or information exchange that is executed using any information and communications technology". According to the nature of the transactions, the following types of e-commerce are distinguished: business-tobusiness (B2B), business-to consumer (B2C), consumer-to consumer $(\mathrm{C} 2 \mathrm{C})$, consumer-to-business $(\mathrm{C} 2 \mathrm{~B})$, no business ecommerce (use of the Internet by nonbusiness organizations such as academic institutions or government agencies to reduce expenses or improve services), and intra-business ecommerce.

E-Commerce systems provide both commercial information (such as products prices and available quantities) and facilitate various commercial actions (e.g., buying, selling and negotiation). The increasing use of information technology in this area has led to fundamental changes in the way these commercial activities are (e.g., the rise of dynamic pricing, the ability to easily compare many goods and ability to negotiate contracts much more frequently) and as more advanced information processing techniques are brought to bear so the potential for further changes increases [3].
E-commerce models are still in the early stages of exploration and applications. There are Some problems which related with e-commerce models such as, platform security, technical standards, regulatory and other services are not well resolved yet in practice, However with the advent of cloud computing, can solve many of these problems.

Cloud computing has been one of the most booming technology among the professional of information technology and also the business due to its elasticity in the space occupation and also the better support for the software and the infrastructure. It attracts more technology specialist towards it. Cloud plays the vital role in the smart economy, and the possible regulatory changes required in implementing better applications by using the potential of cloud computing [4].

Cloud computing can be defined as "A style of computing where massively scalable information technology related capabilities are provided as a service across the internet to multiple external customers"[5]. It is an evolution from distributed computing system, consisting of collection of inter-connected and virtualized computers that provide services dynamically as one or more unified computing resources based on service level agreements (SLA).

The main advantage of cloud computing is the new technology that it gives the low cost implementation for infrastructure (hardware, Software and license for all) and some higher business units like google and Microsoft which offer the cloud for free of cost for the education systems and business application. The cloud computing as anew service model, with network storage, on-demand access to nature, provides a new information resource sharing and processing mechanisms. In the existing conditions, cloud computing framework allows enterprises with less investment to ecommerce business applications (B2B \& B2C). Currently, the combination of e-commerce and cloud computing research focuses on the technical level, therefore, cloud computing based e-commerce application framework will have a high practical value $[6,7]$.

Many business companies do not have the resources and infrastructure needed to run top e-commerce solution. Usually, e-commerce systems are developed as distributed applications, but this is not necessary so. The architecture of a distributed e-commerce system includes software components, like the client application, an application server and database server (see fig. 1) and the necessary hardware components (client computer, communication infrastructure and servers $[8,9,10]$. 


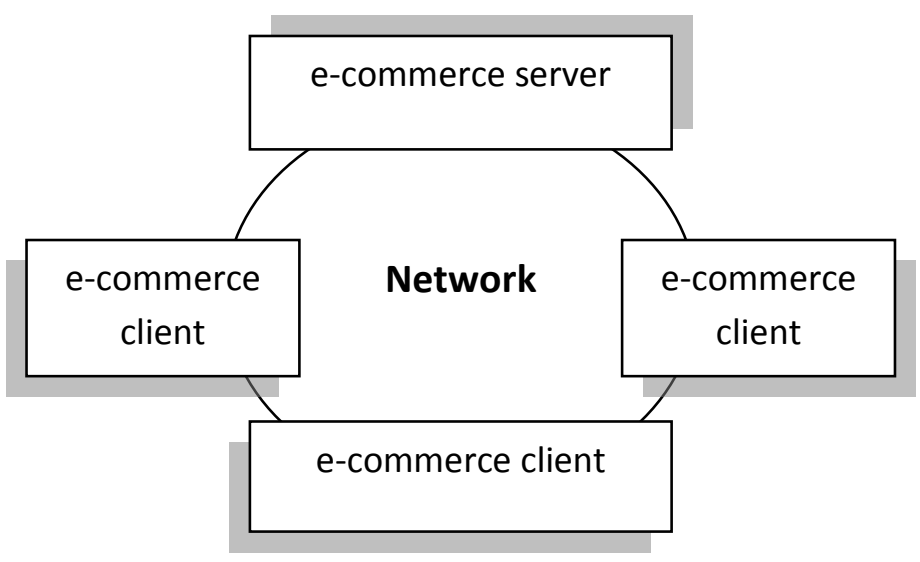

Fig .1 : e-commerce system [10].

The client hardware could be a mobile device or a desktop computer. The client application can be a simple web browser or a dedicated application. The e-commerce server will use cloud computing, so all the required resources will be adjusted as needed [10].

E-commerce systems can use benefit from cloud computing using [6]:

- Infrastructure: use an e-commerce solution on the provider's infrastructure.

- Platform: use the develop an e-commerce solution based on the providers' development interface.

- Services: use the e-commerce solution given by the provider.

Cloud computing is widely accepted today due to its key advantages [10]:

1. The cost is low. Also there are no costs (or very small ones) for hardware upgrades;

2. Flexibility: Infrastructure can be scaled to maximize investments. Cloud computing allows dynamic scalability as demands fluctuate.

3. Accessibility: This help makes data and services publicly available without make vulnerable sensitive problem.

4. devices with minimal hardware requirements (mobile phones, for example) cloud be successfully used as cloud clients.

5. In order to become part of the cloud, there is no need to download or install specific software, only the internet connection is required.

6. Crash recovery is nearly unneeded . If the client computer crashes, there are almost more data lost because everything is stored into the cloud.

In Addition to the previous advantages, there are some disadvantages of the cloud computing [6]:

1. The internet connection speed may affect the over all performances.

2. On along term basis, the data center subscription fee may be more expensive than buying the hardware.

3. The service quality is crucial and the need of the backups is critical when speaking about data security.
The cloud computing provides some major security benefits for individuals and companies that are using/developing ecommerce solutions, like the following:

- Improved improbability.

- Virtualization

- Centralized data storage.

- Monitoring of data access.

\section{Related Work:}

Cloud computing has drawn significant attention from researchers in the past several years. For example, Wang [11] proposed a system that improves the e-commerce in the cloud computing environment. He constructed of new model for the problem exploration. He also parsed related issues on ecommerce development model based on cloud computing. His main aim was to propose the basic idea of building new ebusiness models based on cloud computing, and exploration of related issues as well as the appropriate resolution of ideological and describe how to use the power of cloud computing to open up e-commerce to develop a new pattern.

Liu [12] introduced the e-commerce application model based on cloud computing and coped with the problem of ecommerce and the shortage of resources by establishing the framework of e-commerce application based on the environment of cloud computing service model. He tried to solve the problems of the shortage of funds, man power and technology which were required in the activities of ecommerce. His studies had shown that, in enterprises and their own e-commerce through the network's resources. Cloudbased e-commerce application model allows enterprises to lower costs through the effective implementation of ecommerce activities, and solves the problem of enterprises' can not develop e-commerce activities due to lack of resources.

Jie [13] tried to solve the problem of the transaction security in $\mathrm{C} 2 \mathrm{C}$ E-commerce. A valuation model was proposed based on cloud model theory. By means of using the algorithm of the merger of the trust cloud and the similarity computing of the trust cloud, sub-attribute valuation and comprehensive valuation is achieved. According to the valuation, the two side of transaction got strategic advices. Experiments showed that the method of valuation model was practical and valid. This model was not only applied to $\mathrm{C} 2 \mathrm{C}$ E-commerce, but also a valuable method was provided for trust valuation in opened network.

Sun and Yuan [14] developed an Online Selling Syndicates system with Spring framework and several Apache open source projects for this market based on Software as a service (SaaS) model and analysis of customers' requirements, multichannel and multi-tenant are supported by the system. For convert IT resource to business concept, a controller cluster mode was designed to decentralize the statue control of cloud computing. Two fast check algorithms were designed to serve the efficient control.

Armbrust et al. [15] provided a comprehensive overview of cloud computing, including the new opportunities it enables, the potential obstacles to its adoption, and a classification of cloud providers.

Juncai and Shao[16] showed that the application of cloud technology in the business field. They introduced the development trend of cloud computing to solve a great 
problem of environmental costs during the enterprises apply the e-commerce. On the other hand, from the characteristics of clouds, they analyzed the network security problems and solutions.

\section{A PROPOSED E-COMMERCE FRAMEWORK BASED ON CLOUD COMPUTING:}

The proposed cloud based e-commerce framework is mainly divided into five layers: (1) Hardware resource layer (2) Software resource layer (3) Resource management layer (4) Server layer and (5) business layer. Figure 2 show the architecture of the proposed framework. In the sub-sequent sections, the main components of the proposed system will be discussed in more detail.

\subsection{Hardware Resource Layer:}

This is the bottom most layer in the cloud service middleware. It is the most important for the total infrastructure of the system. The physical hardware layer is virtualized to provide a flexible adaptive platform to improve resource utilization. The keys to new enterprise data center infrastructure services are the next two layers, the virtualization environment and management layer. The combination of the last two layers ensures that resources in a data center are efficiently managed and can be provisioned, deployed, and configured rapidly. With the help of virtualization, physical servers, network and storage are grouped and called it as upper software platform. To offer the uninterruptible power to the cloud middleware servers for the cloud based e-commerce systems, physical host pool is expanded dynamically and memory is scalable at any time to add additional memory.

\subsection{Software resource layer:}

The second layer is created to communicate the operating systems and middleware technology. Many of these software solutions combine to offer the grouped interface for the software developers. Therefore, software developers can create many applications for e-commerce system and able to embed those in cloud, which helps the cloud users to compute these application through cloud.

\subsection{Resource Management layer:}

This layer plays an important role in managing the last two layers. It get loose coupling of software and hardware resources. With the help of virtualization and scheduling idea of cloud computing, it brings the uninterrupted on demand software distribution for different hardware resources.

\subsection{Service Layer:}

This layer is divided into three sub-levels namely IAAS, PAAS and SAAS. These service layers help to cloud customers to use the various forms of cloud resources for their products like software resource, hardware resource, and Infrastructure resource.

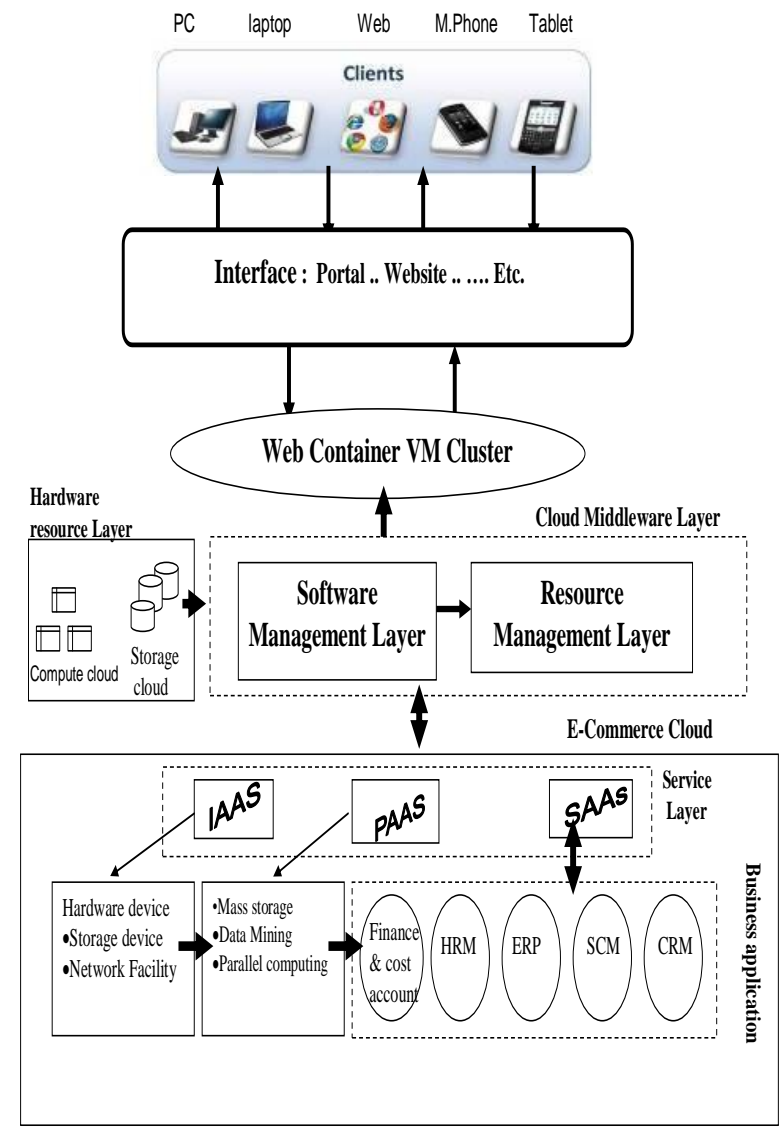

Fig. 2: A Proposed e-Commerce Framework based on cloud computing.

\subsubsection{Infrastructure as a service (Iaas) of e- commerce cloud:}

This layer is the base layer of e-commerce cloud shares IT infrastructure resources and connects the huge system pool together to provide services. Cloud computing allows the hardware layer to run more like the internet, to make the hardware resources shared and accessed as data resources in secure and scalable way. Virtualization technology separates the physical hardware from operating system, which on one hand can make computing and storage capacity of the existing server into smaller size and re-integration, to improve the utilization and flexibility of IT resource; on the other hand can provide a common interface for large-scale cloud computing integration that enables the publication of calculation. The base layer can provide the basic hardware resources for the platform layer, and the users can also make use of it as the same as using a local device to use.

\subsubsection{The platform as a service layer of $e$ - commerce cloud:}

With the support of the powerful hardware, platform layer carries out the tasks of data storage, computing and software development. It can even achieve the tasks of completion of the original mass data storage, business intelligence processing and so on which have been difficult to complete. Users can choose the devices and the number of devices according to the complexity of dealing with the content. Virtualization technology enables the platform to show a strong level of flexibility. 


\subsubsection{Software as a service (SAAS) of $e$ - commerce cloud:}

The applications software or services provided by a professional company e-commerce, the companies to pay in the similar way of on-demand access, according to the amount to calculate the cost, complete the production, marketing, trading and management. Companies use e-commerce system in lower cost to avoid wasting, and more resources can be used for business activities.

E-commerce cloud environment provides user-oriented ubiquitous adaptive hardware resources, computing environment and software services. In e-commerce cloud space, users can access to digital services transparently at any time in anywhere. The users can obtain the necessary network and computing services very naturally at any position. The information space and physical space will be integrated because of ubiquitous computing capability. And the ubiquitous information terminals together with the embedded system equipment will be the vehicles of ecommerce in the future.

\subsection{Business application Layer:}

Business application layer differs from all other layers in cloud based e-commerce architecture, because this layer acts as important business logic of e-commerce, and frames the expansion of group of components for e-commerce. There are growing number of SAAS services which could provide different aspects of capability enabling business to leverage cloud platform to run and manage business processes such as : sales tracking management, goods creation and evaluation, customer relationship management (CRM) , Self Serves Customer Portal, Supply chain management (SCM), Finance and Cost accounting, Enterprise resource management (ERP),Human resource management (HRM)..Etc.

Thus, cloud solution saves us from having to purchase, maintain and train IT staff on expensive hardware and proprietary software code. With One Network's technology solutions in the cloud, we no longer have to purchase, maintain, update and replace expensive equipment. The cloud solution also enables us to provide industry-leading time-tovalue, with implementation times averaging less than half the time of traditional supply chain solutions. In addition, realtime data across all of your value chain participants means increased revenue opportunities. So, say goodbye to costly equipment, maintenance and proprietary programming languages. And get acquainted with instant data flow across your entire value network. Connect to your entire value network in the cloud and see how easy it is to lower your costs and increase your profits.

\section{THE IMPORTANCE OF INTEGRATION BETWEEN CLOUD COMPUTING AND E- COMMERCE:}

There are a set of factor which related with the progress of EC development, it needs are changing into its constraints because of the limitation of enterprise size , economic strength, and technical force, which is mainly showed in following aspects $[17,18]$ :

1. Technical problems such as mass data storage, data mining, Information security .. etc., become a tough set, especially for small and medium-sized e-commercial businesses.
2. Growing equipment \&operation cost are bound to make troubles in the development of e-commerce system.

3. Limitation of information processing capacity and safety performance is imperfect, all of which impede the development of e-commerce.

Nevertheless, with the emergence of cloud computing and the progress of technology it is provides for e-commerce good opportunity to develop, which can be showed as follows:

1. E-Commerce based on cloud computing (e-commerce cloud) environment provide large scale of data center in which mass data storage, high-speed computation, and data mining capacity and significant cost advantage to develop e-commerce business.

2. In e-commerce cloud model, data storage is highly distributed, data management is highly centralized and data service is highly virtualization, all of which offer a much secure data service.

3. Because the e-commerce cloud environment reduces the demand of access to the terminal, the problems of information processing, transmitting and security can be solved neatly.

4. Operation and management based on cloud computing. Ecommerce business can process data flexibility, minimize the operation cost, and realize the automation of solutions in application without considering the position of equipment resources [17].

5. Supply Chain Management (SCM) based on the cloud computing. Cloud computing offers secure and reliable, service of data storage and calculation in time whenever clients need [17].

\section{MAJOR PROBLEMS OF E- COMMERCE CLOUD:}

Cloud computing is a new practice mode applied to ecommerce. The integration of cloud computing and ecommerce has not yet reached a mature stage and still needs the test of practice. At present, there are still many problems that need to be solved $[\mathbf{1 8}, \mathbf{1 9}, \mathbf{2 0}]$ :

\subsection{Security Issues of Cloud Computing Platforms:}

Cloud security includes the data security and the confidentiality of privacy. Currently, all types of cloud computing, i.e., private cloud, public cloud, mixed clouds, and other concepts, have been proposed and gradually applied to practice, but whether they are effective remains to be further verified.

\subsection{Challenges of Cloud Applications:}

For some e-commerce companies, entrusting the work to the third party contains some elements of risks. These risks may be greater than the benefits for the business. Therefore, they will be worried to developed their system with a connect to a third parity.

\subsection{The Standards of Cloud Computing:}

The cloud-based e-service model is still in a fragmented state. If the users really want to promote and apply these new models, a unified industry standard should be developed. 


\subsection{Regulatory Issues of Cloud Services}

There are many services which can be provided through the cloud computing platforms. Information processing, data storage, security, maintenance and other work are usually deal with by the cloud service providers in cloud computing environment. Then, the service provider's position will become crucial to properly handle the information that is related to the user's information security. Therefore, there is an important issue is how to monitor service.

\section{CONCLUSION:}

At present, there is a great problem of environmental cost during the enterprises apply the e-commerce. Therefore, coming of cloud computing all of the problems will be solved in the enterprise e-commerce application. This article introduced a proposed e-commerce framework based on cloud computing. On the other hand, these e-commerce models are still in the early stages of exploration and applications. Some problems such as Analyzing network security, technical standards, regulatory and other services are not well resolved yet in practice, pending further research and exploration. Either way, e- commerce application model based on cloud computing will not stop its pace to proceed. As the cloud computing technologies become more sophisticated and the applications of cloud computing become increasingly widespread, e-commerce will certainly usher in a new era of cloud computing.

\section{REFERENCE}

[1] Till, "Transforming the Way We Do Business," Electronic Commerce, T. Nash, ed., pp. 9-12, 1998.

[2] Electronic Commerce: A Managerial Perspective, E. Turban, J. Lee,D. King, and H.M. Chung, eds. Prentice Hall, 1999.

[3] M.J. Shaw, "Electronic Commerce: State of Art," Handbook on Electronic Commerce, M. Shaw, R. Blanning, T. Strader, and A. Whinston, eds., chapter 1, pp. 3-24, Springer, 2001.

[4] Bcigalupo, D; Wills, G; De Rouse D ; victor, Acategorization of cloud computing business models: IEEE/ACM, May 2010.

[5] Head,M., and Hassanein,k., “ Trust in e-commerce: Evaluating the impact of third-party seals", Quarterly journal of e-commerce, 2002.

[6] C.L.Li, Z.H. Deng., on the value of cloud computing,Library and Information , No.4, 2009.

[7] Yan wang and kwei-jay lin., "Reputation Oriented Trustworthly computing in e-commerce environments", 2007
[8] Boja C., Bataganl., Software characteristics of MLearning application, WSEAS International conference on mathematics, March23-29,2009.

[9] Vetrici M., Improving software project quality using criticality analysis, proceedings of the ninth international conference on informatics in economy IE 2009, Bucharest, Romania.

[10] Paul. P, Felician A, Marius V., " Measuring the efficency of cloud computing for e-learning systems ", WSEAS transactions on computers, Issue1, Volume9, Jan 2010.

[11] Xiaofeng Wang, "Research on e-commerce development model in the cloud computing environment", System Science and Engineering (ICSSE), International Conference on June 30 2012-2 July, 2012.

[12] Tairan Liu ., "E-Commerce Application Model Based on Cloud Computing", Information Technology, Computer Engineering and Management Sciences (ICM), ,International Conference on 24-25 Sept. 2011

[13] Zhang Jie., "Trust evaluation model based on cloud model for $\mathrm{C} 2 \mathrm{C}$ electronic commerce" Computer Application and System Modeling (ICCASM), International Conference on 22-24 Oct. 2010.

[14] Jiangyu Sun; Chun Yuan.,"A multi-channel Online selling Syndicates based on cloud structure",Consumer electronics,Communications and Networks (CECNet),2012 2nd International Conference on 2012.

[15] M. Armbrust, A. Fox, R. Griffith, A.D. Joseph, R.H Katz, A. Konwinski, G. Lee, D. Patterson, A. Rabkin, I. Stoica, and Others. Above the clouds: A berkeley view of cloud computing. EECS Department, University of California,Berkeley, Tech. Rep. UCB/EECS-2009-28, 2009.

[16] Shen Juncai and Qian Shao.,"Based on Cloud Computing E-Commerce Models and Its Security", International journal of e-education, e-Business,e-Management and elearningVol1,No.2. June,2011.

[17] Gunasekar. K, Anirudh . C., " Analysis of security issues in cloud based e-learning " master's thesis in informatics university of boras, Aug 2011

[18] Tairon Liu, E-commerce application model based on cloud computing, International conference of information technology, computer engineering and management sciences, IEEE,2011

[19] M. Tim, K. Subra, L. Shahed. Cloud Security and Privacy: An Enterprise Perspective on Risks and Compliance. O'Reilly Media, Inc., 2009

[20] S. Dan, C. Roger. Privacy and consumer risks in cloud computing. Computer law and security review, vol.26, pp.391-397, 2010 